\title{
Coupling MicroNIR/Chemometrics for the on-site detection of cannabinoids in hemp flours
}

\author{
Roberta Risolutia ${ }^{,{ }^{*}}$, Giuseppina Gullifaa ${ }^{a}$, Alfredo Battistini ${ }^{\mathrm{b}}$ and Stefano Materazzi ${ }^{\mathrm{a}}$ \\ aDepartment of Chemistry - “Sapienza” University of Rome, p.le A.Moro 5, 00185 Rome Italy \\ b Consiglio per la ricerca in agricoltura e l'analisi dell'economia agraria - Centro di Politiche e Bioeconomia, via Pò 14, \\ 00198 Italy \\ * Correspondence: roberta.risoluti@uniroma1.it; Tel.:+390649913616; Fax: +390649387137
}

\begin{abstract}
In this work, the capabilities of a novel miniaturized and portable MicroNIR spectrometer were investigated in order to propose a practical and intelligible test allowing the rapid and easy screening of cannabinoids in hemp flour. In order to develop a predictive model able to identify and simultaneously to quantify the residual amount of cannabinoids, specimens from flours commercially available on the markets were considered and spiked with increasing amount of Cannabidiol (CBD), $\Delta^{9}$-Tetrahydrocannabinol (THC) and Cannabigerol (CBG). Partial Least Square-Discriminant Analysis (PLS-DA) and Partial Least Square regression (PLSr) were applied for the simultaneously detection and quantification of cannabinoids. Results demonstrated that MicroNIR/Chemometric platform is statistically able to identify the presence of CBD, THC and CBG in simulated samples containing cannabinoids from 0.001 to $0.1 \% \mathrm{ww}$, with the accuracy and sensitivity of the reference official methods actually proposed. The method was checked against false positive and true positive response and results proved to be those required for confirmatory analyses; permitting to provide a fast and accurate method for the monitoring of cannabinoids in hemp flours.
\end{abstract}

Keywords: cannabinoids; hemp flour; MicroNIR; chemometrics

\section{Introduction}

Hemp based products has been recently considered as promising materials originating from cannabis and containing numerous natural compounds with medicinal properties [1,2] such as flavonoids, mono and sesqui-terpenes, steroids, nitrogenous compounds and cannabinoids, an exclusive class of characteristic structures (C21 terpenophenolics) [3-5]. The distribution of more than 70 cannabinoids [6] makes the hemp plant one of the most attractive and monitored matrix from law enforcement and food vendors. In fact, among these, the psychotropic THC represents the cannabinoid with the highest affinity to cannabinoid receptors (CB1, CB2) [7,8]. In addition, despite cannabidiol (CBD) and cannabigerol (CBG) do not exhibit CB binding affinity, these non psychotropic molecules are frequently investigated due to their interesting and effective properties for medicinal uses [9-11]. As a consequence, there is a growing concern associated to the monitoring of cannabinoids in hemp based products especially when used for nutraceutical or food purposes.

Regardless to confirmatory analyses, the most common approaches consist of chromatographic methods, including High Performance Liquid Chromatography followed by Mass Spectrometry (HPLC-MS) [12-15] and Gas Chromatography coupled to Mass Spectrometry (GC-MS) [16-18].

In the meanwhile, spectroscopic techniques proved to be promising in characterizing complex matrices and offered the advantages to be easy-to-use and non destructive without requiring any pretreatment [19-21]. In addition, the coupling with chemometric tools provides models of prediction for qualitative as well as quantitative response with the required accuracy as the interference of the matrix in the spectral signal may be evaluated [22-25].

Recently, Near Infrared spectroscopy was considered for the evaluation of the growing stage of cannabis cultivated greenhouse [26]. Despite the advances in the field, all the actually available devices lack of accuracy and do not permit to achieve the suitable sensitivity to avoid true positive and false positive response. As a consequence, miniaturized and one-touch devices providing the identification and quantification of cannabinoids in a completely automated and accurate platform, are more and more required [27, 28]. 
In this work, a novel analytical platform based on MicroNIR spectroscopy associated to chemometric analysis is proposed for a fast and accurate detection of cannabinoids in hemp flours. The main advantage of this compact system is related to the miniaturized portable spectrometer operating in the Near Infrared region that permits to obtain comparable outcomes as the laboratory one because of its geometry and optical resolution [29-34]. In addition, the association with chemometrics provided an automated and non destructive tool able to simultaneously detect and quantify traces of cannabinoids in complex matrices and permitted to further investigate the same sample with the reference official procedure (GC-MS) for confirmatory analyses.

\section{Material and methods}

\subsection{Materials and samples collection}

Reference standard solution of cannabidiol (CBD), $\Delta^{9}$-Tetrahydrocannabinol (THC) and cannabigerol (CBG) were purchased from Sigma-Aldrich (St. Louis, Missouri United States) as methanolic solution at the concentration of $1 \mathrm{mg} / \mathrm{ml}$ and all the standards were pure at $99 \%$.

With the aim of calibrating the spectral response and to evaluate the matrix effect on cannabinoids identification, a preliminary step was performed by the mean of simulated samples. To this end, 10 different hemp flours commercially available in the markets (cannabinoids free) were analyzed as such and spiked with CBD, THC and CBG by the mean of a rotary evaporator. A number of 5 spectra were recorded for each specimen (about $1 \mathrm{mg}$ ) as replicated, in order to ensure heterogeneity of materials and the response being not dependant of the different flour.

No sample pretreatment was required for MicroNIR measurements and the hemp flours were directly processed by the platform. On the other hands, a proper clean-up was put in place for the same samples prior to GC-MS analysis in order to accomplish the confirmatory analyses.

\subsection{MicroNIR platform}

A portable spectrometer platform MicroNIR, developed and distributed by Viavi Solutions (JDSU Corporation, Milpitas, USA) and operating in the spectral region 900-1700 nm was used for spectra collection. This ultra-compact and low-cost device consist of a linear variable filter (LVF) as dispersing element directly connected to a 128-pixel linear indium gallium arsenide (InGaAs) array detector and two tungsten light bulbs as radiation source. The MicroNIR is equipped with a special collar to get the optimum focal point of the illumination from the spectrometer's window to the sample to be measured.

Collection of spectra was performed at a nominal spectral resolution of $6.25 \mathrm{~nm}$. The MicroNIR spectrometer used a NIR reflectance standard (blank), with a 99\% diffuse reflectance (Spectralon) while a dark reference was obtained from a fixed place in the room. The acquisitions were performed with an integration time of $10 \mathrm{~ms}$, resulting in a total measurement time of $2.5 \mathrm{~s}$ per sample. The MicroNIR Pro software (JDSU Corporation, Milpitas, USA) was employed for MicroNIR instrument control and all chemometric treatments were carried out by V-JDSU Unscrambler Lite (Camo software AS, Oslo, Norway).

Principal Component Analysis (PCA) has been used as unsupervised technique to evaluate whether the approach MicroNIR/Chemometrics may differentiate samples according to different cannabinoids, thus discriminating the original hemp seeds as CBD, THC or CBG type. To this end, 10 different specimens were considered for each molecule and increasing amounts of cannabinoids in the range 0.001 to $0.1 \%$ ww were added to estimate the cannabinoids content.

Three regression models were developed by Partial Least Square (PLS) regression for the prediction of CBD, THC and CBG in hemp flour and a comprehensive model was also developed in order to simultaneously identify the three main cannabinoids usually present in hemp. In addition, PLS regression was used to quantify the CBD, THC and CBG content, in order to simultaneously quantify the psychoactive molecule.

As recommended for spectroscopic data [39], mathematical pre-treatments were considered for chemometric evaluation: in particular, among scatter-correction methods, Standard Normal Variate transform (SNV), [35] Multiplicative Scatter Correction (MSC) and Mean Centering (MC) [36] and normalization [37] were evaluated, while Savitzky-Golay (SG) polynomial derivative filters [38] was considered as spectral derivation techniques. 


\subsection{GC-MS Confirmatory analyses}

Confirmatory analyses were performed on all the investigated samples in order to check the platform performences. The GC-MS system was a Perkin Elmer (Waltham, MA) interfaced with a mass selective detector, using a HP-5MS ( $30 \mathrm{~m} \times 0.25 \mathrm{~mm} \times 0.25 \mathrm{~mm}$ ) as capillary separation column and operating according to reference official procedures. The carrier gas was helium delivered at a constant flow of $1 \mathrm{~mL} / \mathrm{min}$. The oven temperature program was initially $120^{\circ} \mathrm{C}$ for $1 \mathrm{~min}$, ramped to $240^{\circ} \mathrm{C}$ at $30^{\circ} \mathrm{C} / \mathrm{min}$ and then increased to $290^{\circ} \mathrm{C}$ at $10^{\circ} \mathrm{C} / \mathrm{min}$ for $10 \mathrm{~min}$, with a post run was made at $300{ }^{\circ} \mathrm{C}$ for $5 \mathrm{~min}$. Electron impact (EI) ionization was employed at a voltage of $70 \mathrm{eV}$. The interface temperature was $250^{\circ} \mathrm{C}$, and the temperature of the ion source and quadrupole were $230^{\circ} \mathrm{C}$ and $150^{\circ} \mathrm{C}$, respectively. Mass spectral data was collected in the scan mode from $\mathrm{m} / \mathrm{z} 44$ to 450 while Selected Ion Monitoring (SIM) mode was used for the identification of analytes. The injection was made in split mode with a split ratio of 50:1 [40].

\section{Results and discussion}

The platform is entirely designed and developed with the aim of simplifying controls of hemp food products and to facilitate the rapid interpretation of results, as once the model is assessed, it can be used to process real samples. All the experiments were carried out focusing on two main issues: the development of a model of prediction as robust as possible; the variability of the spectroscopic signal as a function of the matrix effect of different hemp flours and as function of the added cannabinoid. In fact, as the type of hemp plant changes, the residual content of cannabinoids results in a different distribution of CBD, THC and CBG in the flours. To this aim, the interferences on the spectral signal due to the complexity of the matrix were considered by evaluating spectra of reference standard solutions of cannabinoids and spectra of 10 different spiked hemp flours. This procedure was necessary to ensure the results being independent of the different composition of flours and permitted to evaluate the ability of the approach to detect cannabinoids in complex matrices. This is the main advantage of the coupling approach of chemometrics to MicroNIR analysis: in fact, the multiparametric tools may help in interpreting results of complex matrices by selecting the most descriptor region of the spectra, reducing noise and enhancing the contribution of the analyte with respect to interferences.

Spectra in the range 900-1700 $\mathrm{nm}$ were recorded and the typical profile of the reference standards of CBD (red line), THC (blue line) and CBG (green lines) is reported in Figure 1a, while the collected spectra of hemp flours fortified with CBD, THC and CBG are overlapped in Figure 1b. 
$1 a$
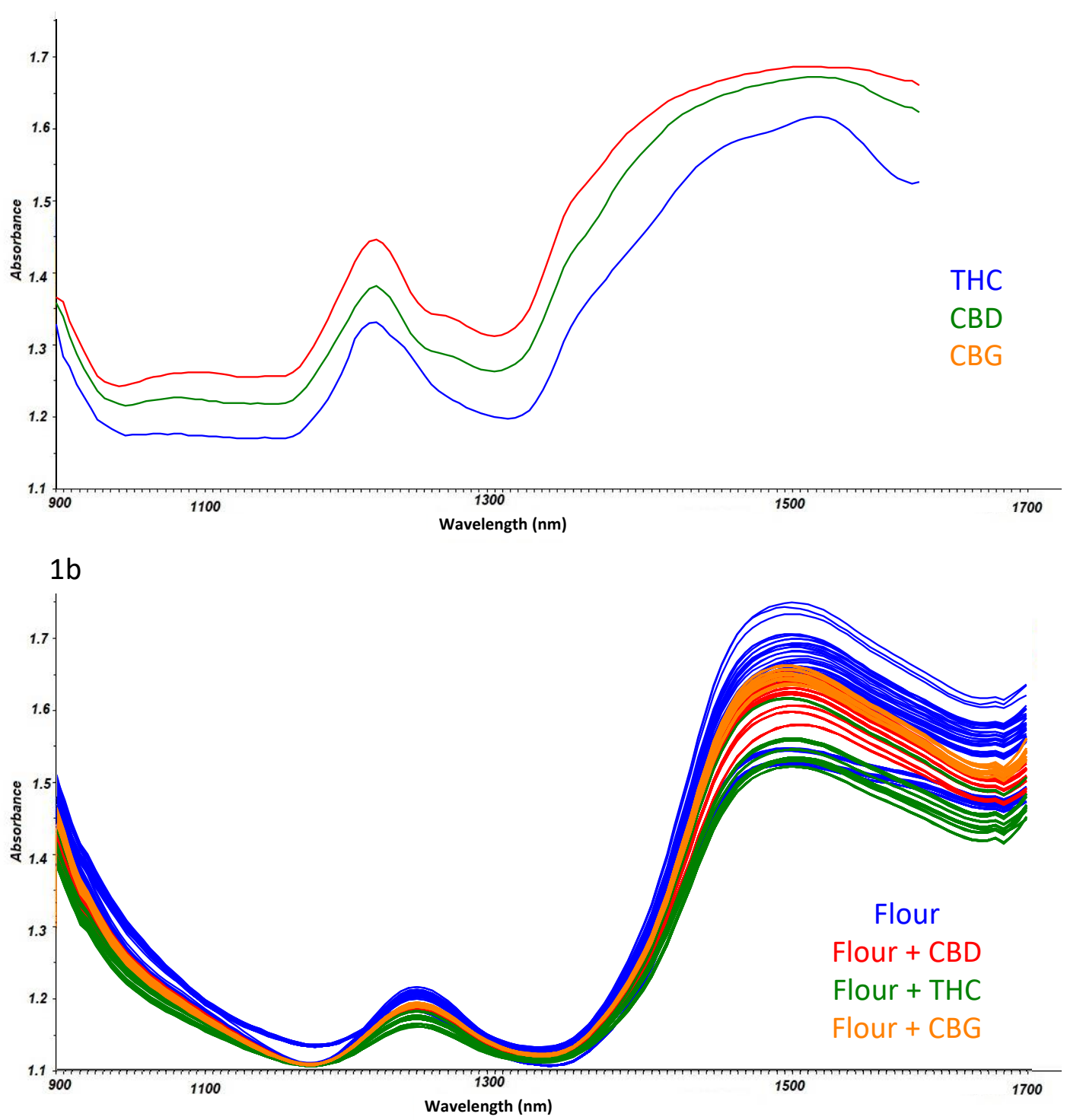

Figure 1. MicroNIR spectra of a) the reference standards of CBD (red line), THC (blue line) and CBG (green lines); b) flour (blue) and fortified flours with CBD (red line), THC (blue line) and CBG (green lines).

Figure 1 reveals a significant matrix effect due to the complexity and the heterogeneity of the hemp flour specimens. As a consequence, Principal Component Analysis (PCA) was applied to the recorded spectra in order to select the diagnostic variables reducing the contribution of the matrix as not all the wavelengths contribute equally to samples separation.

Different chemometric spectral pretreatments were investigated in order to provide the best separation of samples as a function of the presence or the absence of cannabinoids, such as Standard Normal Variate (SNV), Mean Centering (MC) transform, multiplicative scatter correction (MSC) and normalization [35-37]; in addition, the Savitzky-Golay (SG) polynomial derivative filter [38] was also considered as spectral derivation technique. Among these, baseline correction followed by first derivative transform and Mean Centering provided the best separation of all the processed spectra for CBD samples when the range of wavelengths 1110-1323 nm was selected, as reported in Figure 2a. Regardless to THC and CBG, the most performing results were obtained when spectra were baseline corrected and followed by MC pre-treatments, as shown in Figure $2 \mathrm{~b}$ and Figure $2 \mathrm{c}$. These procedures permitted to distinguish signals apparently overlapped into different classes as reported in the scores plots in Figure 2. 

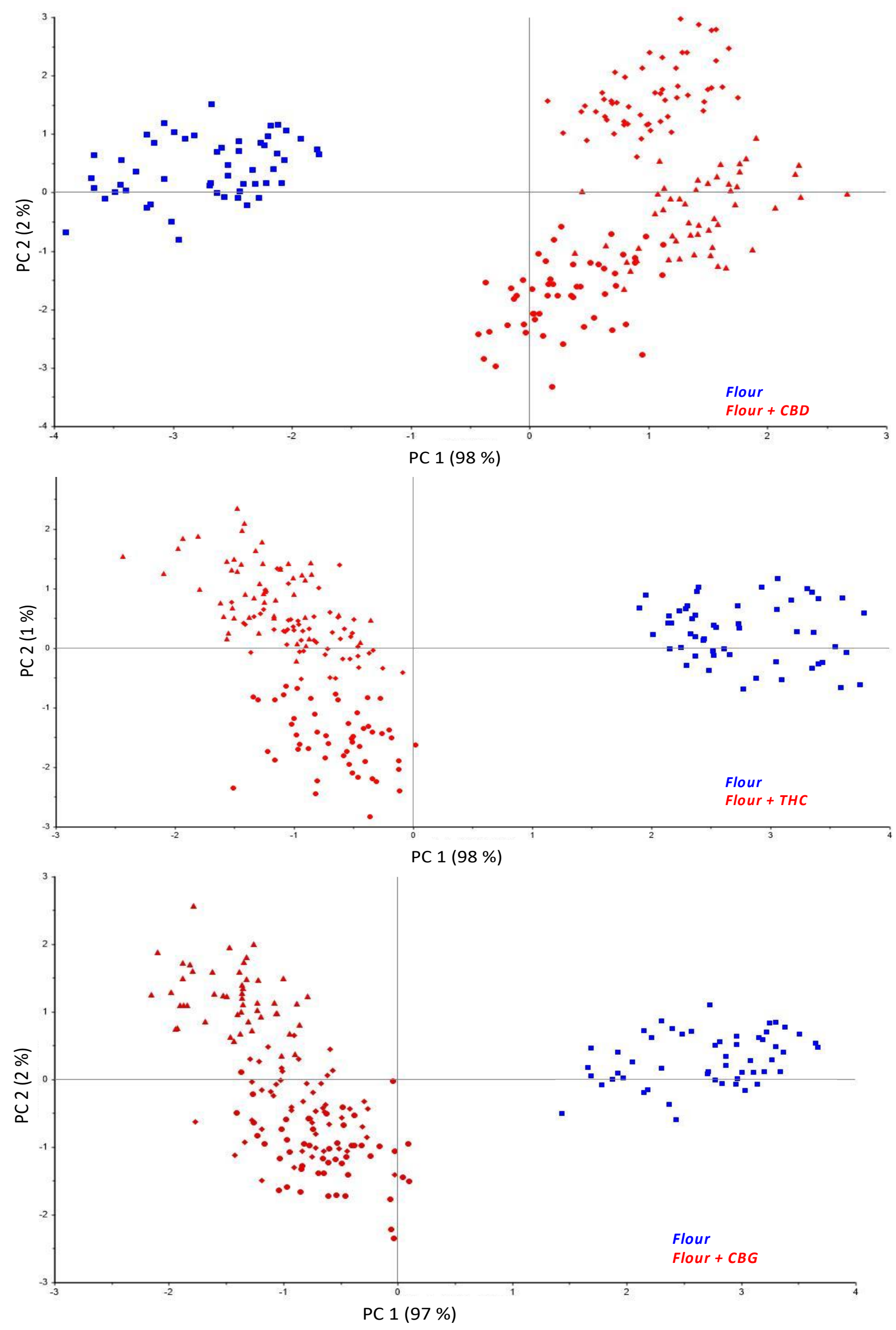
Figure 2. Resulting scores plot by PCA of hemp flours (blue) and spiked hemp flours CBD (a), THC (b) and CBG (c).

Despite the different involved molecules, all the investigated samples were found to be located in different side of the plots according to PC 1 that explains an overall variance of $98 \%$ for CBD, THC and CBG.

Further experiments were performed in order to develop a tool able to simultaneously predict the three main cannabinoids in hemp flours to be used for the monitoring of the hemp food products. Samples were previously investigated by Principal Component Analysis that confirmed the suitable separation of the samples according to PC 1 (overall explained variance of $79 \%$ ) as a consequence of the different molecule recovered in the samples, as reported in Figure 3.

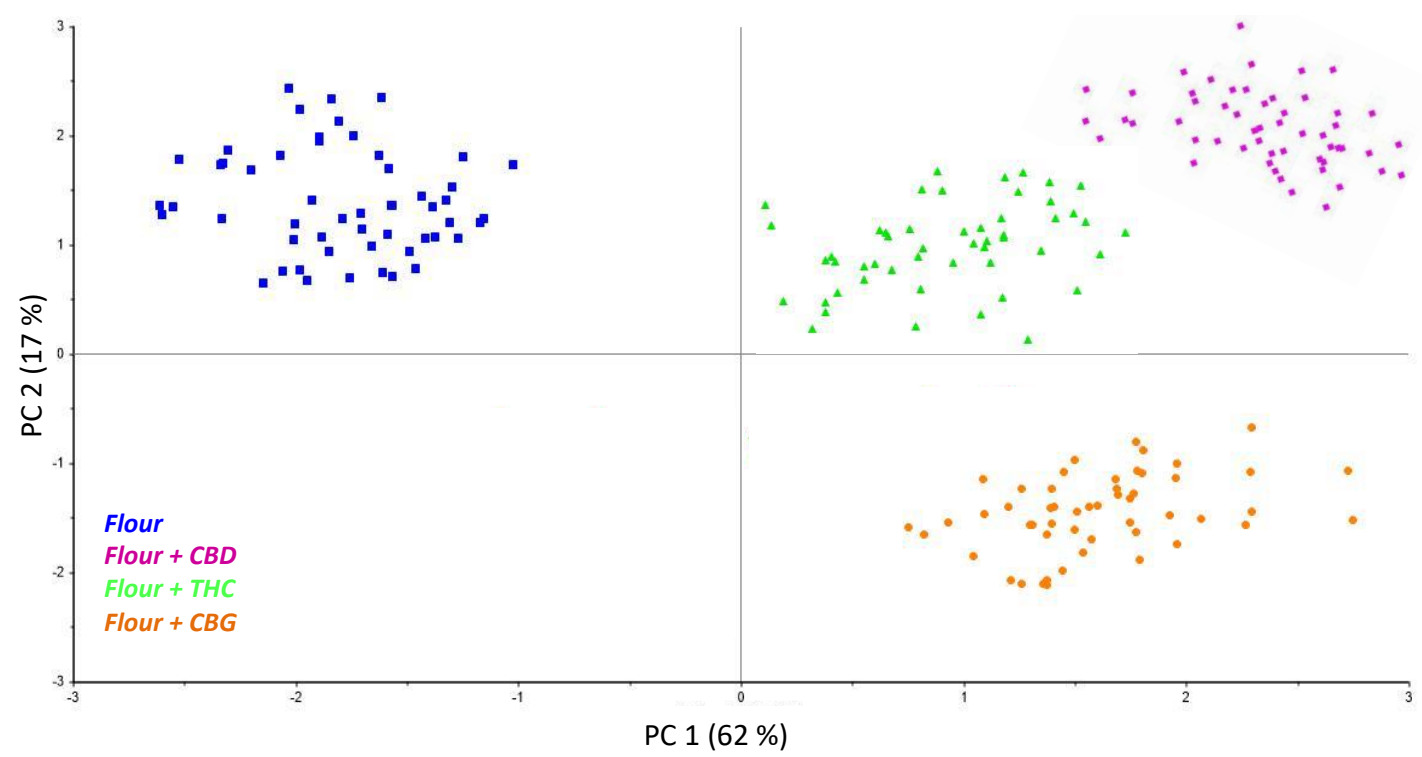

Figure 3. Resulting scores plot by PCA of flour (blue) and fortified flours with CBD (pink), THC (light green) and CBG (orange).

Consequently, a model of prediction of the cannabinoid content was developed by Partial Least SquareDiscriminant Analysis (PLS-DA) and its performances were evaluated by estimating the figures of merit such as Non Error Rate (NER \%), Specificity (Sp\%) and Root Mean Square Error of calibration (RMSEC), validation (RMSECV), and prediction (RMSEP). All the spectra were baseline corrected and the first derivative transform followed by MC was used in the range 1100-1617 $\mathrm{nm}$. Satisfactory calibration results were obtained as reported in Table 1: the model provided for an accuracy and a specificity not lower than $100 \%$ and an error of prediction not exceeding $0.009 \%$ both in calibration and validation.

Table 1. Figures of merits obtained by PLS-DA model for cannabinoids in hemp flours.

\begin{tabular}{ccccccccc}
\hline & \multicolumn{3}{c}{ Calibration } & & \multicolumn{3}{c}{ Prediction } \\
\hline & & Blank & Blank & Blank & & Blank & Blank & Blank \\
& Blank & + & + & + & Blank & + & + & + \\
& & CBD & THC & CBG & & CBD & THC & CBG \\
\hline NER (\%) & 100 & 100 & 100 & 100 & 100 & 100 & 100 & 100 \\
Sp (\%) & 100 & 100 & 100 & 100 & 100 & 100 & 100 & 100 \\
RMSE & 0.0001 & 0.002 & 0.001 & 0.002 & 0.0001 & 0.002 & 0.002 & 0.004 \\
\hline
\end{tabular}




\subsection{Quantification method}

This behaviour suggested the possibility to further investigate whether the MicroNIR/Chemometric approach may differentiate samples according to the quantity of the cannabinoid.

A chemometric model was developed for the prediction of the residual cannabinoids content in hemp flours, based on Partial Least Square regressions (PLSr) considering a maximun of 6 latent variables. The collected spectra were divided into calibration set (about $75 \%$ of the dataset) and validation set (about $25 \%$ of the collected spectra), while the prediction ability of the developed methods was evaluated considering the determination coefficient $\left(\mathrm{R}^{2}\right)$, the Root Mean Squared Errors (RMSEs), the slope and offset in calibration and validation. Results are reported in Table 2 . In addition, to estimate the model's performances, the precision and the sensitivity were also determined in order to provide a tool able to simultaneously detect the presence and the amount of each cannabinoid.

Table 2. Prediction ability of the method

\begin{tabular}{ccccccccc}
\hline \multicolumn{3}{c}{ Calibration } & \multicolumn{5}{c}{ Prediction } \\
\hline & Slope & offset & RMSE & R-square & Slope & offset & RMSE & R-square \\
\hline CBD & 0.9877 & 0.0008 & 0.004 & 0.9876 & 0.9620 & 0.0030 & 0.006 & 0.9741 \\
THC & 0.9981 & $1.102 \mathrm{e}-04$ & 0.002 & 0.9981 & 0.9973 & $1.594 \mathrm{e}-04$ & 0.002 & 0.9980 \\
CBG & 0.9900 & 0.0060 & 0.004 & 0.9900 & 0.9757 & 0.0014 & 0.004 & 0.9873 \\
\hline
\end{tabular}

Suitable results were achieved for each molecule, since the correlation values were always higher than 0.988 in calibration and not lower than 0.974 in validation. In addition, the model for THC prediction provided the most performing values of RMSE $(0.002 \% w w)$, with respect to CBD $(0.06 \% w w)$ and CBG $(0.004 \% w w)$ models.

A further significant outcome consists of the performances of the models that demonstrated to be sensitive (about $0.10 \%$ for CBD and $0.2 \%$ for THC and CBG) and precise (about $1.23 \%, 1.56 \%$ and $1.12 \%$ for $\mathrm{CBD}$, THC and CBG respectively) enough to ensure a fast and accurate detection to be used for forensic purposes.

\subsection{Predicted versus reference outcomes}

All the prepared samples were simultaneously processed by the reference method (GC-MS) and the MicroNIR platform and the percentage of the cannabinoids recovered in the portion of flour analyzed by MicroNIR light source was compared to that obtained by the extraction of $500 \mathrm{mg}$ of hemp flour for GC-MS analysis. A good correlation among the data from the two methods was observed since all the samples were correctly predicted by the model and the correlation coefficient resulting from the analysis of the predicted (GC-MS outcomes) versus measured (MicroNIR measurements) samples was about 0.989 .

\section{Conclusions}

A novel screening system based on a MicroNIR/Chemometric platform, able to perform the first level test of cannabinoids in hemp flour was developed and validated. The platform was entirely designed by reproducing real caseworks and with the aim of simplify the monitoring of hemp food products with accuracy and sensitivity of the confirmatory methods. A model of prediction based on PLS regression was developed for each molecules taken into account the three main cannabinoids such as CBD, THC and CBG. In addition, a comprehensive PLS-DA model was pointed out with the aim of simultaneously detecting and differentiating the three molecules. All the samples were simultaneously processed by the reference official method in order to check the prediction ability of the models. Results provided for a method allowing a non destructive, 
solvent-less and easy way to perform a fast and accurate identification of cannabinoids. In addition, the coupling MicroNIR/Chemometric ensured the automation of the platform that was entirely designed with the aim of simplify measurements and to facilitate the rapid interpretation of results, as once the model is assessed, it can be used to process real samples in a "click-on" device.

The limit of detection and accuracy of the method were found to be those required for confirmatory tests according to law regulations, concluding that the MicroNIR approach permits to obtain comparable performances as the reference procedures and to simplify the monitoring of feeds in order to ensure the quality of veterinary supplements.

Conflicts of Interest: The authors have declared no conflict of interest

\section{References}

1. Aizpurua-Olaizola, O.; Omar, J.; Navarro, P.; Olivares, M.; Etxebarria, N.; Usobiaga, A. Identification and quantification of cannabinoids in Cannabis sativa L. plants by high performance liquid chromatography-mass spectrometry. Anal. Bioanal. Chem. 2014, 406, 7549-7560.

2. Romano, L.L.; Hazekamp, A. Cannabis Oil: chemical evaluation of an upcoming cannabis-based medicine. Cannabinoids 2013, $1(1), 11$.

3. Werz, O.; Seegers, J.; Schaible, A.M.; Weinigel, C.; Barz, D.; Koeberle, A.; Allegrone, G.; Pollastro, F.; Zampieri, L.; Grassi, G.; Appendino, G. Cannflavins from hemp sprouts, a novel cannabinoid-free hemp food product, target microsomal prostaglandin E 2 synthase-1 and 5-lipoxygenase. Pharma Nutrition 2014, 2, 53-60.

4. ElSohly, M.A.; Slade, D. Chemical constituents of marijuana: the complex mixture of natural cannabinoids. Life Sci. 2005, $78,539-548$.

5. Radwan, M.M.; ElSohly, M.A.; El-Alfy, A.T.; Ahmed, S.A.; Slade, D.; Husni, A.S.; Manly, S.P.; Wilson, L.; Seale, S.; Cutler, S.J.; Ross, S.A.J. Isolation and Pharmacological Evaluation of Minor Cannabinoids from High-Potency Cannabis sativa. Nat. Prod. 2015, 78, 1271-1276.

6. Peschel, W.; Politi, M. ${ }^{1} \mathrm{H}$ NMR and HPLC/DAD for Cannabis sativa L. chemotype distinction, extract profiling and specification. Talanta 2015, 140, 150-165

7. Mechoulam, R.; Hanus, L.. A historical overview of chemical research on cannabinoids. Chem. Phys. Lipids 2000, 108, 113.

8. Grotenthermen, F. Curr. Drug Targets CNS Neurol. Disord. 2005, 4, 507-530.

9. Esposito, G.; De Filippis, D.; Maiuri, M.C.; De Stefano, D.; Carnuccio, R.; Iuvone, T. Cannabidiol inhibits inducible nitric oxide synthase protein expression and nitric oxide production in beta-amyloid stimulated PC12 neurons through p38 MAP kinase and NF-kappaB involvement. Neurosci. Lett. 2006, 399, 91-95.

10. Pertwee, R.G. The diverse CB1 and CB2 receptor pharmacology of three plant cannabinoids: $\Delta 9$-tetrahydrocannabinol, cannabidiol and $\Delta$ 9-tetrahydrocannabivarin. Br. J. Pharmacol. 2008, $15,199-215$.

11. Borrelli, F.; Fasolino, I.; Romano, B.; Capasso, R.; Maiello, F.; Coppola, Orlando, P.; Battista, G.; Pagano, E.; Di Marzo, V.; Izzo, A.A. Beneficial effect of the non-psychotropic plant cannabinoid cannabigerol on experimental inflammatory bowel disease. Biochem. Pharmacol. 2013, 85, 1306-1316.

12. Hadener, M.; Konig, S.; Weinmann, W. Quantitative determination of CBD and THC and their acid precursors in confiscated cannabis samples by HPLC-DAD. Forensic Science International. 2019, https://doi.org/10.1016/j.forsciint.2019.03.046

13. Ciolino, L.A.; Ranieri, T.L.; Taylor, A.M. Commercial cannabis consumer products part 2: HPLC-DAD quantitative analysis of cannabis cannabinoids. Forensic Science International 2018, 289, 438-447

14. Elkins, A.C.; Deseo, M.A.; Rochfort, S.; Ezernieks, V.; Spangenberg, G. Development of a validated method for the qualitative and quantitative analysis of cannabinoids in plant biomass and medicinal cannabis resin extracts obtained by super-critical fluid extraction. Journal of Chromatography B 2019, 1109, 76-83

15. Patel, B.; Wene, D.; Fan, Z.T. Qualitative and quantitative measurement of cannabinoids in cannabis using modified HPLC/DAD method. Journal of Pharmaceutical and Biomedical Analysis 2017, 146, 15-23 
16. Pellegrini, M.; Marchei, E.; Pacifici, R.; Pichini, S. A rapid and simple procedure for the determination of cannabinoids in hemp food products by gas chromatography-mass spectrometry. Journal of Pharmaceutical and Biomedical Analysis 2005, 36, 939-946

17. Cardenia, V.; Toschi, T.G.; Scappini, S.; Rubino, R.C.; Rodriguez-Estrada, M.T. Development and validation of a Fast gas chromatography/mass spectrometry method for the determination of cannabinoids in Cannabis sativa L. Journal of food and drug analysis 2018, 26, 1283-1292

18. Burnier, C.; Esseiva, P.; Roussel, C. Quantification of THC in Cannabis plants by fast-HPLC-DAD: A promising T method for routine analyses. Talanta 2019, 192, 135-141

19. Véstia, J.; Barros, J.M.; Ferreira, H.; Gaspar, L.; Rato, A.E. Predicting calcium in grape must and base wine by FT-NIR Spectroscopy. Food Chemistry 2019, 276, 71-76

20. Materazzi, S.; Risoluti, R.; Pinci, S.; Romolo, F.S. New insights in forensic chemistry: NIR/Chemometrics analysis of toners for questioned documents examination. Talanta 2017, 174, 673-678.

21. Oliveri, P.; Di Egidio, V.; Woodcock, T.; Downey, G. Application of class-modelling techniques to near infrared data for food authentication purposes. Food Chemistry 2011, 125, 1450-1456. 22. Meesa, C.; Souard, F.; Delported, C.; Deconinck, E.; Stoffelen, P.; Stévigny, C.; Kauffmann, J.F.; De Braekeleer, K. Identification of coffee leaves using FTNIR spectroscopy and SIMCA. Talanta 2018, 177, 4-11.

23. Risoluti, R.; Materazzi, S.; Gregori, A.; Ripani, L. Early detection of emerging street drugs by near infrared spectroscopy and chemometrics. Talanta 2016, 153, 407-413.

24. Materazzi, S.; Peluso, G.; Ripani, L.,; Risoluti, R. High-throughput prediction of AKB48 in emerging illicit products by NIR spectroscopy and chemometrics. Microchemical Journal 2017, 134, 277-283.

25. Kordi, B.; Kovacevi, M.; Slobod, T.; Vidovi, A., Jovi, B. FT-IR and NIR spectroscopic investigation of hydrogen bonding in indole-ether systems. Journal of Molecular Structure 2017, 1144, 159-165.

26. Borille, B.T.; Caetano, C.; Marcelo, A.; Scorsatto Ortiz, R.; de Cássia Mariotti, K.; Flôres Ferrão, M.; Pereira Limberger, R. Near infrared spectroscopy combined with chemometrics for growth stage classification of cannabis cultivated in a greenhouse from seized seeds. Spectrochimica Acta Part A: Molecular and Biomolecular Spectroscopy 2017, 173, 318-323.

27. Houwing, S.; Hagenzieker, M.; Mathijssen, R.P.M.; Legrand, S.A.; Verstraete, A.G., Hels, T., Bernhoft, I.M., Simonsen, K.W., Lillsunde, P., Favretto, D., Ferrara, S.D.; Caplinskiene, M.; Movig, K.L.L.; Brookhuis, K.A. Random and systematic errors in case-control studies calculating the injury risk of driving under the influence of psychoactive substances. Accident Analysis and Prevention 2013, 52, 144-153.

28. Risoluti, R.; Gregori, A.; Schiavone, S.; Materazzi, S. “Click and Screen" technology for explosives detection on human hands by portable MicroNIR/Chemometrics platform. Analytical Chemistry 2018, 90, 4288-4292.

29. Paiva, E.M.; Rohwedder, J.J.R.; Pasquini, C.; Pimentel, M.F.; Pereira, C.F. Quantification of biodiesel and adulteration with vegetable oils in diesel/biodiesel blends using portable near-infrared spectrometer. Fuel 2015, 160, 57-63.

30. Modrono, S.; Soldado, A.; Martínez-Fernández, A., de la Roza Delgado, B. Handheld NIRS sensors for routine compound feed quality control: Real time analysis and field monitoring. Talanta 2017, 162, 597-603.

31. Risoluti, R.; Materazzi, S.; Tau, F., Russo, A.; Romolo, F.S. Towards innovation in paper dating: a MicroNIR analytical platform and chemometrics. Analyst 2018, 143, 4394-4399.

32. Risoluti, R.; Materazzi, S. MicroNIR/Chemometrics assessement of occupational exposure to hydroxyurea. Frontiers in Chemistry 2018, 6, 228.

33. Basri, K.N.; Hussain, M.N.; Bakar, J.; Khir, M.F.A., Zoolfakar, A.S. Classification and quantification of palm oil adulteration via portable NIR spectroscopy. Spectrochim Acta A, Mol Biomol Spectrosc. 2017, 173, 335-342.

34. da Silva, N.C.; Cavalcanti, C.J.; Honorato, F.A.; Amigo, J.M.; Pimentel, M.F. Standardization from a benchtop to a handheld NIR spectrometer using mathematically mixed NIR spectra to determine fuel quality parameters. Analytica Chimica Acta 2017, 954, 32-42.

35. Barnes, R.J.; Dhanoa, M.S.; Lister, S.J. Standard Normal Variate Transformation and Detrending of Near-Infrared Diffuse Reflectance Spectra. Appl. Spectros. 1989, 43, 772-777.

36. Geladi, P.; MacDougall, D.; Martens, H. Linearization and scatter-correction for Near-Infrared reflectance spectra of meat. Appl. Spectrosc. 1985, 39, 491-500.

37. Wold, S.; Sjöström, M. Chemometrics: Theory and Applications. In: B.R. Kowalski (Ed.), American Chemical Society Symposium Series 1977, 52, 243-282A.

38. Savitzky, M.J.; Golay, E. Smoothing and differentiation of data by simplified least squares procedures. Anal.Chem. 1964, 
36, 1627-1639.

39. Rinnan, A.; van den Berg, F.; Engelsen, S.B. Review of the most common pre-processing techniques for near-infrared spectra. Trends in Analytical Chemistry, 2009, 28, 10.

40. Pichini, S.; Pacifici, R. Linee guida per la determinazione di sostanza d'abuso nella saliva, Istituto Superiore di Sanità, 2013. 